Service social

\title{
Les impacts de l'informatisation des données de service social en milieu hospitalier
}

\section{Georgette Béliveau, Martin Poulin et Ginette Beaudoin}

Volume 42, numéro 2, 1993

Recherche et pensée critique

URI : https://id.erudit.org/iderudit/706620ar

DOI : https://doi.org/10.7202/706620ar

Aller au sommaire du numéro

\section{Éditeur(s)}

École de service social de l'Université Laval

ISSN

1708-1734 (numérique)

Découvrir la revue

Citer cet article

Béliveau, G., Poulin, M. \& Beaudoin, G. (1993). Les impacts de l'informatisation des données de service social en milieu hospitalier. Service social, 42(2),

103-126. https://doi.org/10.7202/706620ar
Résumé de l'article

Cet article fait suite à un premier paru dans la même revue en 1992. Les deux écrits rapportent les résultats d'une recherche portant sur l'informatisation des données de service social en milieu hospitalier. Le premier article faisait état d'observations liées au processus d'implantation, tandis que le second traite de l'impact de l'informatisation. Il s'agit d'une étude longitudinale descriptive à caractère exploratoire faite dans six cliniques d'un hôpital. Le bilan de l'expérience est tantôt qualifié de positif, tantôt de formateur. Il ressort de la recherche que la concertation entre les gestionnaires et les intervenants en service social s'impose comme modèle à privilégier à partir de la première étape du processus, à plus forte raison lorsqu'il s'agit d'implanter un projet d'informatisation portant sur un contenu d'intervention professionnelle. Bien que plusieurs impacts positifs aient été observés, on peut croire qu'au moment où les intervenants des cliniques disposeront d'ordinateurs, d'autres changements de plus grande importance surviendront. 


\section{Les impacts de l'informatisation des données de service social en milieu hospitalier}

Georgette BÉLIVEAU Professeure titulaire, École de service social Université Laval

Martin POULIN Professeur titulaire, École de service social Université Laval

Ginette BEAUDOIN Professionnelle de recherche École de service social Université Laval

L'informatique dans les services sociaux a d'abord été introduite pour supporter les systèmes $d^{\prime}$ 'information de gestion. Elle connaît depuis une diffusion plus généralisée et une plus grande diversification des champs d'application. Ces dernières années, on voit poindre certaines applications à la pratique professionnelle'. "À cet égard, le processus d'informatisation des données de service social engagé à l'hôpital Sainte-Justine de Montréal constitue un bon

1. Pour une meilleure vue d'ensemble de l'évolution, le lecteur peut se reporter aux ouvrages antérieurs des auteurs cités dans les références bibliographiques ci-après: Béliveau, Poulin et Beaudoin (1991 et 1987); Poulin, Beaudoin, Béliveau et al. (1991); Poulin et Béliveau (1987). 
exemple d'une nouvelle application technologique à la pratique du service social. En effet, deux intervenantes sociales rattachées à cet établissement, Françoise Turgeon-Krawczuk et Claire Lalande-Gendreau, ont conçu un logiciel d'intervention familiale et sociale visant à informatiser les données de service social et à les intégrer aux données médicales. L'originalité de cette démarche et les effets engendrés par une telle entreprise ont suscité notre intérêt en tant que professionnels et chercheurs en service social. Nous avons donc accepté l'invitation des deux intervenantes d'accompagner l'expérimentation par un projet de recherche ${ }^{2}$ qui s'est échelonné sur trois ans (juin 1988 à juin 1991) grâce à une subvention du Conseil de la recherche en sciences humaines du Canada. La recherche touchait les volets de l'implantation de l'ordinateur et celui des impacts reliés à cette implantation.

Un article paru dans la revue Service social ${ }^{3}$ (1992) faisait état des observations liées au processus d'implantation, alors que le présent article traite de l'impact. Il s'agit d'une étude longitudinale descriptive à caractère exploratoire. Cette étude de cas, de nature qualitative, vise principalement à décrire les impacts de l'informatisation selon certaines dimensions. Elle ne suppose pas l'élaboration d'un cadre théorique traditionnel ni d'hypothèses de départ. Nous avons suivi la méthode de travail suggérée par Yin (1984) pour élaborer ce type de recherche. Nous y reviendrons plus en détail au moment d'expliquer le processus d'analyse.

La première partie du présent article traite des dimensions de l'étude, alors que la seconde porte sur la collecte et l'analyse des données. Les sections suivantes exposent les impacts dans les différentes cliniques à l'étude, alors qu'une autre section donne la vision des gestionnaires. Nous concluons enfin par des observations plus générales.

\section{LES DIMENSIONS DE L'IMPACT DE L'INFORMATISATION}

La conception que nous avons privilégiée pour l'étude des « impacts de l'informatisation » s'apparente de près à celle de Danziger et al. (1987), Larsen (1987) et Hamelin-Brabant (1987). Selon ces auteurs, les conséquences de I'informatisation ne sont pas unidimensionnelles et facilement visibles; elles se manifestent plutôt de manières variées, subtiles et évoluent dans le temps,

2. G. Béliveau, M. Poulin et G. Beaudoin (1992), "L'implantation d'un système informatisé des dossiers de service social en milieu hospitalier», Service social, vol. $41, \mathrm{n}^{\circ} 1: 104$ et 105 .

3. Ibid. : 104-126. 
d'où la difficulté d'en saisir toute l'ampleur. L'informatisation peut donc susciter des changements positifs, négatifs et pluridimensionnels, qui peuvent être prévus, alors que d'autres ne sont pas anticipés par les membres de I'organisation. Les impacts de l'informatisation peuvent être très variés et il est nécessaire de préciser la façon dont ils ont été définis dans le cadre de cette recherche. L'objectif général de la recherche est de définir les impacts de l'informatisation dans le milieu de travail. C'est ainsi qu'on a recueilli des informations factuelles et des données subjectives regroupées sous deux volets: la «description» comprend les indicateurs portant sur ce qui se passe dans le milieu de travail ; le volet " perception » précise les changements perçus par les personnes engagées dans le processus d'informatisation. À partir de la recension des écrits et en tenant compte de l'interprétation du milieu dans lequel s'inscrit cette étude, la description des dimensions et la perception du changement ont porté sur l'accomplissement de la tâche, la pratique professionnelle, les communications, la confidentialité, le contrôle et l'évaluation, l'image professionnelle des intervenants et les craintes et attentes. Le choix des dimensions à l'étude est inspiré des écrits sur le sujet. L'articulation des sous-dimensions est aussi tirée des mêmes sources avec un souci particulier d'adaptation à la réalité locale. Enfin, les indicateurs ont pu être précisés grâce à la collaboration d'un informateur clé issu du milieu. Cette façon de procéder donne un caractère tantôt plus théorique, tantôt plus pratique à la description et à l'opérationalisation des dimensions, mais elle a l'avantage de refléter le contexte dans lequel s'inscrit la recherche.

\section{L'accomplissement de la tâche}

Le tableau qui suit décrit les principales tâches ou les fonctions des praticiens, des médecins-directeurs des cliniques, du personnel administratif ou des coordonnateurs et des gestionnaires. Cette description permet de préciser la répartition des tâches dans l'organisation. Puisque les tâches effectuées par le personnel peuvent être affectées par l'informatisation, il est bon de vérifier, comme la littérature le suggère, si l'on perçoit des changements dans les tâches elles-mêmes, dans leur diversité, dans la charge et le rythme du travail et dans l'autonomie professionnelle des personnes.

La seconde sous-dimension, "utilisation de l'ordinateur», porte sur les personnes qui font usage de l'ordinateur, la nature du travail effectué avec celui-ci, la fréquence d'utilisation, la formation reçue en vue de son utilisation, I'assistance et l'aide disponibles. La dernière sous-dimension traite de la description de l'environnement physique de l'ordinateur: son emplacement, son fonctionnement et l'aménagement physique de son environnement. 


\section{TABLEAU 1}

\section{Dimension : accomplissement de la tâche}

\begin{tabular}{|c|c|c|}
\hline & Sous-dimensions & Indicateurs \\
\hline \multirow[t]{2}{*}{ 1) } & $\begin{array}{l}\text { Répartition } \\
\text { des tâches }\end{array}$ & $\begin{array}{l}\text { Description des tâches ou fonctions principales: } \\
\text { a) des intervenants sociaux } \\
\text { b) du personnel administratif et coordonnateur } \\
\text { c) des médecins } \\
\text { d) des gestionnaires }\end{array}$ \\
\hline & & $\begin{array}{l}\text { Perception des changements dans: } \\
\text { a) les tâches } \\
\text { b) la charge de travail } \\
\text { c) le rythme de travail } \\
\text { d) la diversité du travail } \\
\text { e) l'autonomie au travail }\end{array}$ \\
\hline 2) & $\begin{array}{l}\text { Utilisation } \\
\text { de I'ordinateur }\end{array}$ & $\begin{array}{l}\text { Description: } \\
\text { a) des personnes qui font usage de l'ordinateur } \\
\text { b) de la nature du travail effectué } \\
\text { c) de la fréquence d'utilisation } \\
\text { d) de la formation que possèdent les utilisateurs } \\
\text { e) de l'assistance ou l'aide disponible }\end{array}$ \\
\hline 3) & $\begin{array}{r}\text { Environnement } \\
\text { physique de } \\
\text { travail }\end{array}$ & $\begin{array}{l}\text { Description: } \\
\text { a) de l'emplacement de l'ordinateur } \\
\text { b) du fonctionnement de l'ordinateur } \\
\text { c) de l'aménagement physique }\end{array}$ \\
\hline
\end{tabular}

\section{La pratique professionnelle}

La pratique professionnelle se définit comme étant l'ensemble des activités directes et indirectes des intervenants reliées à leur intervention auprès des clients. Les quatre sous-dimensions étudiées sont: le contenu et la tenue des dossiers, le suivi professionnel du bénéficiaire et le cheminement des dossiers.

En procédant à l'informatisation des dossiers des bénéficiaires, le contenu et la tenue des dossiers risquent d'être passablement modifiés. La première sous-dimension, le contenu des dossiers, est étudiée sous les angles du type de données, de la nature des documents contenus dans le dossier, de leur uniformité et de leur caractère facultatif.

La tenue des dossiers, deuxième sous-dimension, est liée au mode d'entrée des informations, c'est à-dire aux formes sous lesquelles sont colligées les informations et aux formats sous lesquels on les retrouve dans les dossiers. Il est possible qu'une clinique rendue à un stage intermédiaire dans le processus d'informatisation possède les mêmes données, en partie dans les dossiers traditionnels et en partie sur disquettes. Il se peut aussi que des informations différentes se retrouvent dans I'un ou l'autre format. Ces informations visent à 


\section{TAbleau 2}

\section{Dimension : pratique professionnelle}

\begin{tabular}{|c|c|c|}
\hline & Sous-dimensions & Indicateurs \\
\hline 1) & $\begin{array}{r}\text { Contenu } \\
\text { des dossiers }\end{array}$ & $\begin{array}{l}\text { Description: } \\
\text { a) du type d'information } \\
\text { b) du type de document } \\
\text { c) des documents ou informations uniformes } \\
\text { dans tous les dossiers } \\
\text { d) des documents facultatifs aux dossiers } \\
\text { e) autres aspects }\end{array}$ \\
\hline 2) & $\begin{array}{r}\text { Tenue } \\
\text { des dossiers }\end{array}$ & $\begin{array}{l}\text { Description: } \\
\text { a) de la forme de collecte des informations } \\
\text { (forme narrative, grille, notes, tests, codes) } \\
\text { b) du format (dossiers traditionnels } \\
\text { ou sur disquettes) } \\
\text { c) du contenu et de la fréquence de la mise à jour } \\
\text { Perception des changements dans: } \\
\text { a) la pratique } \\
\text { b) le portrait du bénéficiaire } \\
\text { c) l'utilisation des dossiers } \\
\text { d) I'accès aux dossiers }\end{array}$ \\
\hline 3) & $\begin{array}{r}\text { Suivi } \\
\text { professionnel } \\
\text { du bénéficiaire }\end{array}$ & $\begin{array}{l}\text { Description: } \\
\text { a) du suivi dans la clinique } \\
\text { b) du suivi par le service social } \\
\text { c) du suivi quant au diagnostic, aux révisions, } \\
\text { à la planification } \\
\text { d) de la procédure de prise de rendez-vous } \\
\text { Perception de changements quant: } \\
\text { a) à la qualité de la relation avec le bénéficiaire } \\
\text { b) à la qualité de la prise de décision professionnelle } \\
\text { c) au suivi du bénéficiaire } \\
\text { d) à la procédure de rendez-vous }\end{array}$ \\
\hline 4) & $\begin{array}{r}\text { Cheminement } \\
\text { du dossier } \\
\text { (monitoring } \\
\text { system) }\end{array}$ & $\begin{array}{l}\text { Description: } \\
\text { a) du cheminement des dossiers du service social } \\
\text { dans I'hôpital et au CSS } \\
\text { b) de la politique d'ouverture et de fermeture } \\
\text { des dossiers } \\
\text { c) des données disponibles à l'hôpital, } \\
\text { à la clinique et au CSS } \\
\text { Perception de changements quant à: } \\
\text { a) la disponibilité des données de service social } \\
\text { b) la rapidité de l'acheminement des données } \\
\text { de service social }\end{array}$ \\
\hline
\end{tabular}


mieux connaître comment l'organisation se réajuste à mesure que le processus d'informatisation se poursuit. La tenue des dossiers fait aussi référence au contenu et à la fréquence des mises à jour (mises à jour effectuées de façon automatique, mensuelle, etc.). Au volet descriptif, on a joint les perceptions du personnel concerné quant aux changements survenus dans la pratique professionnelle, dans la description du portrait psychosocial du bénéficiaire et dans l'utilisation des dossiers et l'accès à ces derniers.

La sous-dimension «suivi professionnel du bénéficiaire » se situe au cœur de la pratique professionnelle. Le volet descriptif comprend le suivi du bénéficiaire dans la clinique ou tel que fait par le praticien social et les mécanismes de suivi en rapport avec le diagnostic, les révisions, la planification des interventions et la prise de rendez-vous. La littérature mentionne que l'informatique aura une influence sur la qualité de la relation avec le bénéficiaire et sur la qualité des décisions. On recueille donc les perceptions des praticiens quant aux changements provoqués par l'informatisation relativement à ces deux aspects. La grille laisse place à la possibilité de relever d'autres changements concernant le suivi du bénéficiaire et la procédure de rendez-vous.

La dernière sous-dimension reliée à la pratique professionnelle est d'ordre organisationnel : il s'agit de décrire le cheminement des dossiers du service social à l'intérieur de l'hôpital et du Centre de services sociaux (CSS) ${ }^{4}$, les politiques d'ouverture et de fermeture des dossiers, les données de service social disponibles à la clinique, à l'hôpital et au CSS. Le cheminement des dossiers du service social revêt une importance particulière, car leur informatisation pourrait, selon certains, améliorer la rapidité d'acheminement et les délais de consultation. C'est pourquoi les membres du personnel ont été invités à livrer leurs perceptions des changements intervenus sous ces rapports.

\section{La communication}

La communication se définit comme «l'échange d'information y compris les différentes façons de transmettre et de recevoir les connaissances » (Barker, 1987 : 28) et comme «la transmission formelle ou informelle de messages $d^{\prime}$ 'une personne à l'autre, d'un groupe à un autre en vue de mettre une action en marche ou d'en modifier le cours » (Dion, 1986: 99). Les écrits révèlent que l'informatisation a un impact important sur les communications. On prévoit, par exemple, que les appels aux collègues pour compléter l'information

4. L'étude ayant été faite de juin 1988 à juin 1991, il est normal qu'on conserve dans ce texte l'ancienne terminologie en ce qui a trait aux établissements. De plus, les intervenants sociaux étaient alors engagés par le CSS et prêtés au centre hospitalier par contrat de services. 
seront moins fréquents, puisque chaque intervenant aura toutes les informations nécessaires dans le dossier informatisé.

Tableau 3

Dimension : communication entre les personnes

\begin{aligned} & \hline Sous-dimension Indicateurs \\ & \hline Avec $\begin{array}{l}\text { Description de la communication avec: } \\ \text { a) le personnel de la clinique } \\ \text { b) le personnel du service social } \\ \text { c) les autres membres du personnel de l'hôpital } \\ \\ \text { Perception des changements } \\ \text { dans la communication avec: } \\ \text { a) le personnel de la clinique } \\ \text { b) le personnel du service social }\end{array} \\ &\end{aligned}$

Dans le cadre de la recherche, l'étude de cette dimension se limite aux communications entre les personnes. Dans un premier temps, la tâche principale consiste à décrire les communications formelles et informelles avec le personnel de la clinique et, selon le cas, avec le personnel du service social. On entend par communication formelle celle qui figure à l'agenda de travail du personnel, par exemple les réunions de groupe ou de comité et les entrevues sur rendez-vous. La communication informelle couvre les échanges improvisés entre les personnes en vue de répondre à des besoins particuliers du moment. Dans un deuxième temps, on vérifie si le personnel a perçu des changements dans la communication et dans la qualité de la communication avec le personnel de la clinique et, selon le cas, avec le personnel du service social.

\section{La confidentialité}

La confidentialité se définit comme «la protection de l'information donnée par un client ou celle acquise par un professionnel dans le cours de sa pratique et le droit à la vie privée » (Timms, 1982 : 95). Béliveau et Poulin (1987) distinguent la confidentialité et la sécurité. La première renvoie aux directives administratives de l'organisation, à ses politiques et à ses règles de fonctionnement au sujet de la circulation des informations personnelles que I'organisation détient. La sécurité, par ailleurs, repose sur une procédure particulière et sur des techniques utilisées pour préserver un degré désiré de confidentialité au sujet des informations personnelles. L'organisation doit établir une politique et une procédure de confidentialité concernant la collecte, I'enregistrement et I'utilisation de cette information. Les deux 
sous-dimensions définies dans le cadre de la recherche sont donc la politique de confidentialité et les mécanismes de sécurité utilisés pour protéger les informations concernant les bénéficiaires.

TABLEAU 4

\section{Dimension : confidentialité}

\begin{tabular}{|c|c|c|}
\hline & Sous-dimensions & Indicateurs \\
\hline \multirow[t]{2}{*}{ 1) } & $\begin{array}{l}\text { Politique de } \\
\text { confidentialité }\end{array}$ & $\begin{array}{l}\text { Description: } \\
\text { a) de la nature des politiques } \\
\text { b) des instances qui voient à leur application } \\
\text { c) du degré de connaissance des politiques existantes }\end{array}$ \\
\hline & & $\begin{array}{l}\text { Perception des changements dans: } \\
\text { a) la politique établie } \\
\text { b) l'impact de l'informatisation sur la confidentialité }\end{array}$ \\
\hline \multirow[t]{2}{*}{ 2) } & $\begin{array}{l}\text { Mécanismes } \\
\text { de sécurité }\end{array}$ & $\begin{array}{l}\text { Description: } \\
\text { a) des mécanismes } \\
\text { b) des instances qui voient à leur application } \\
\text { c) des mesures de sécurité employées } \\
\text { individuellement }\end{array}$ \\
\hline & & $\begin{array}{l}\text { Perception des changements dans: } \\
\text { a) la qualité des mécanismes de sécurité en place }\end{array}$ \\
\hline
\end{tabular}

La première sous-dimension décrit la nature de la politique de confidentialité, les instances qui voient à son application et le degré de connaissance de cette politique. La même démarche s'applique pour la description des mesures de sécurité (deuxième sous-dimension) auxquelles s'ajoutent les différentes mesures individuelles prises par le personnel pour assurer la confidentialité. La perception des changements porte sur la politique de confidentialité et son impact, de même que sur la qualité des mécanismes de sécurité.

\section{Le contrôle et l'évaluation}

Dans les écrits sur le sujet, il est principalement question des impacts de I'informatisation sur le contrôle et l'évaluation du personnel. Dans la recherche, cette dimension couvre également le contrôle du travail professionnel sous l'angle de la vérification de la réalisation des actions prévues et de l'atteinte des objectifs. Les sous-dimensions privilégiées sont les politiques et les mécanismes de contrôle et d'évaluation.

La partie descriptive traite de la nature des politiques et des mécanismes de contrôle et d'évaluation, de même que des instances chargées de leur 
application. Comme certains contrôles peuvent s'effectuer même en l'absence de politiques, les membres du personnel sont interrogés sur les mesures individuelles qu'ils adoptent.

TABLEAU 5

Dimension : contrôle et évaluation

\begin{tabular}{|c|c|c|}
\hline & Sous-dimensions & Indicateurs \\
\hline 1) & $\begin{array}{r}\text { Politiques } \\
\text { de contrôle } \\
\text { et d'évaluation }\end{array}$ & $\begin{array}{l}\text { Description: } \\
\text { a) de la nature des politiques } \\
\text { b) des instances qui voient à leur application }\end{array}$ \\
\hline \multirow[t]{2}{*}{ 2) } & $\begin{array}{r}\text { Mécanismes } \\
\text { de contrôle } \\
\text { et d'évaluation }\end{array}$ & $\begin{array}{l}\text { Description: } \\
\text { a) des mécanismes } \\
\text { b) des instances qui voient à leur application } \\
\text { c) des mesures prises par les personnes }\end{array}$ \\
\hline & & $\begin{array}{l}\text { Perception des changements quant à la façon } \\
\text { d'exercer le contrôle ou l'évaluation: } \\
\text { a) des tâches } \\
\text { b) de la pratique professionnelle } \\
\text { c) du suivi du bénéficiaire }\end{array}$ \\
\hline
\end{tabular}

Les écrits mentionnent que le personnel craint de voir une augmentation du contrôle de son travail ou de sa pratique professionnelle à la suite de l'informatisation. Selon Glastonbury (1986), ces craintes sont anticipées, mais pas toujours vécues. Dans le cas qui nous intéresse, les individus sont interrogés sur leur perception du changement introduit par l'informatique dans la façon d'exercer le contrôle et l'évaluation de leurs tâches, de leur pratique professionnelle et du suivi des bénéficiaires.

\section{L'image professionnelle des praticiens}

En raison des rôles respectifs exercés par les différents groupes d'intervenants dans la pratique multidisciplinaire en milieu médical, l'image professionnelle du praticien revêt une importance particulière. Dans le Social Dictionary Work, on définit l'image du travail social par la façon dont les gens jugent la profession de travailleur social, que leur vision soit exacte ou non. Si l'on s'inspire de cette définition, l'image professionnelle du praticien se fonde sur la perception de la profession et du rôle du travailleur social. L'informatisation peut-elle modifier cette perception? Quel est le point de vue des praticiennes des cliniques informatisées? Ont-elles le sentiment que l'informatisation a eu un impact sur la perception que les autres professionnels ont des travailleuses sociales et de leur pratique en milieu hospitalier? Les médecins-directeurs 
de ces cliniques perçoivent-ils différemment le rôle de la travailleuse sociale depuis l'informatisation? Les indicateurs retenus recoupent ces questions.

\section{Les attentes et les craintes}

Plusieurs auteurs se sont penchés sur les attitudes, les résistances et les craintes des individus au moment de l'implantation de l'informatique dans leur milieu de travail. II va sans dire que l'intérêt porté aux réactions personnelles des individus est fort légitime, car le succès de l'implantation de la technologie informatique serait en grande partie attribuable aux facteurs humains. Aussi s'attarde-t-on aux craintes et aux attentes des personnes à l'égard de l'informatisation des données sociales. L'attente se définit comme un état d'esprit subjectif influencé par la capacité d'ajustement de la personne à une situation potentielle qu'elle anticipe, souvent en fonction de son expérience passée. La crainte, quant à elle, se définit comme un sentiment par lequel on redoute quelqu'un ou quelque chose jugé dangereux ou nuisible.

Dans une perspective d'évolution, l'étude s'intéresse aux attentes et aux craintes qui naissent avant l'informatisation et au cours du processus d'informatisation. Les questions visent ainsi à expliquer, s'il y a lieu, comment les attentes et les craintes se sont modifiées pendant le processus d'informatisation.

\section{LA COLLECTE DES DONNÉES ET LE MODE D'ANALYSE}

L'étude des impacts de l'informatisation a été menée auprès du personnel de six cliniques pédiatriques et des gestionnaires ${ }^{5}$ engagés dans le processus d'informatisation. Pour préserver la confidentialité, les cliniques ont été désignées sous le vocable de clinique $A, B, C, D, E$ ou $F^{6}$. Les cliniques $A$ et

5. Des hommes et des femmes gestionnaires ont participé à notre recherche. Pour préserver I'anonymat des personnes, nous avons utilisé le masculin partout.

6. La clinique $A$ assure la surveillance de près de 300 enfants atteints d'une maladie chronique. Ces derniers demandent des soins à long terme et un suivi médical régulier : tests de sang, surveillance du régime alimentaire, du poids, des médicaments. Quatre visites annuelles sont prévues au protocole de soins établi pour chaque enfant. La clinique B, pour sa part, s'occupe des enfants porteurs d'une malformation. Une semaine après la naissance, ces enfants sont dirigés vers la clinique et sont hospitalisés au cours de la première année deux ou trois fois suivant les chirurgies nécessaires. La clinique B intervient auprès de 190 enfants, dont une cinquantaine de nouveaux cas chaque année. La clinique $C$ traite des enfants atteints d'une maladie grave nécessitant des soins spécialisés et des traitements intensifs et palliatifs. Près de 1500 enfants et familles sont suivis à cette clinique. La clinique $\mathrm{D}$ s'occupe d'enfants et de mères infestés par un virus pouvant conduire à différentes formes d'infections. Près de 40 enfants et leur mère sont suivis à la clinique. La clinique $\mathrm{E}$ assume la surveillance de bébés présentant divers problèmes qui 
B sont celles qui ont été informatisées les premières, les cliniques $C$ et $D$ sont celles qui ont amorcé le processus d'informatisation au cours de la recherche, alors que les cliniques $\mathrm{E}$ et $\mathrm{F}$ ne sont pas informatisées. Dans le présent texte, nous parlons de "cliniques informatisées » lorsque nous faisons référence aux cliniques $A$ et $B$, de «cliniques en voie d'informatisation » lorsqu'il s'agit des cliniques $C$ et $D$ et de «cliniques non informatisées » lorsque nous discutons des deux autres, soit $\mathrm{E}$ et $\mathrm{F}$.

L'entrevue structurée a été l'instrument privilégié pour recueillir les informations. Les entrevues ont été menées dans chaque clinique auprès de membres de l'équipe: le médecin responsable de la clinique, l'intervenante sociale, la secrétaire ou l'infirmière-coordonnatrice de la clinique. Nous avons également interrogé la secrétaire participant au projet à l'unité de service social et quatre gestionnaires ou cadres intermédiaires de l'hôpital et du Centre de services sociaux du Montréal métropolitain (CSSMM).

C'est à partir des indicateurs présentés dans la partie précédente qu'ont été élaborés les schémas d'entrevues. Les personnes interrogées ne participant pas toutes également au processus d'informatisation, des schémas d'entrevues propres à chacune des catégories de répondants ont été mis au point. Ils comprennent des questions communes à tous et des questions particulières à la fonction occupée par chaque groupe de répondants. Les schémas d'entrevues se composent de questions ouvertes regroupées selon les dimensions et les sous-dimensions. La collecte des données a eu lieu au trimestre d'hiver de chacune des années où s'est poursuivie l'étude, soit 1989, 1990 et 1991. Le tableau 6 précise les contributions de chaque groupe de participants à la recherche selon le moment de la collecte des données.

Les informations ont été recueillies à trois reprises dans les cas des cliniques $A, B, C$ et $D$. Les cliniques $E$ et $F$ pour leur part n'ont été considérées qu'aux collectes des hivers 1989 et 1991 . On peut tracer un portrait des impacts de l'informatique à trois moments différents pour les cliniques $\mathrm{A}$ et B. Dans le cas des cliniques $C$ et $D$, qui s'informatisaient plus tardivement, les collectes ont permis de connaître leur fonctionnement avant et pendant leur informatisation. Pour ce qui est des cliniques E et $F$, qui n'envisageaient pas de s'informatiser pendant la période couverte par la recherche, le but était de connaître leur fonctionnement et leurs attentes à l'égard de l'informatisation. Toutes les personnes en fonction et libres au moment des collectes de données ont été interrogées.

nécessitent des soins particuliers. Ces enfants sont hospitalisés dès leur naissance et, par la suite, ils sont vus à la clinique, puis à d'autres moments selon les besoins. Enfin, la clinique $\mathrm{F}$ traite principalement les enfants atteints d'épilepsie et ceux ayant des retards de développement, des troubles d'apprentissage et des maux de tête. L'intervenante sociale intervient principalement auprès des enfants hospitalisés. 


\section{TABLEAU 6}

\section{Moments des collectes des données} et personnel participant aux entrevues

\begin{tabular}{|c|c|c|}
\hline Collecte Hiver-89 & Collecte Hiver-90 & Collecte Hiver-91 \\
\hline $\begin{array}{l}\text { Cliniques A, B, C, D, E, F: } \\
\text { médecins chefs, } \\
\text { intervenantes sociales, } \\
\text { secrétaire / infirmière- } \\
\text { coordonnatrice }\end{array}$ & $\begin{array}{l}\text { Cliniques A, B, C, D: } \\
\text { médecins chefs, } \\
\text { intervenantes sociales, } \\
\text { secrétaire / infirmière- } \\
\text { coordonnatrice }\end{array}$ & $\begin{array}{l}\text { Cliniques A, B, C, D, E, F: } \\
\text { médecins chefs, } \\
\text { intervenantes sociales, } \\
\text { secrétaire / infirmière- } \\
\text { coordonnatrice }\end{array}$ \\
\hline $\begin{array}{l}\text { secrétaire } \\
\text { du service social }\end{array}$ & $\begin{array}{l}\text { secrétaire } \\
\text { du service social }\end{array}$ & $\begin{array}{l}\text { secrétaire } \\
\text { du service social }\end{array}$ \\
\hline 4 gestionnaires & 1 gestionnaire & 4 gestionnaires \\
\hline
\end{tabular}

Nous appuyant sur le modèle proposé par Yin (1984), la stratégie générale d'analyse repose sur le développement d'un cadre conceptuel de description de cas $^{7}$. L'analyse des impacts de l'informatisation se fait en plusieurs étapes, plus ou moins longues, selon le degré d'informatisation des cliniques.

Pour l'analyse des impacts dans les cliniques $\mathrm{A}, \mathrm{B}, \mathrm{C}$ et D, la première étape consiste à regrouper les données selon les dimensions préalablement établies (pattern-matching). L'étape suivante sert à construire un modèle explicatif (explanation-building) des données recueillies. Ce modèle met en relief les ressemblances et les différences en rapport avec ce qui est avancé dans la littérature et fournit l'explication du «comment » et du "pourquoi » des phénomènes observés. Finalement, la dernière étape tente d'élaborer et d'établir les interrelations entre les résultats obtenus d'une clinique à l'autre en retenant leurs ressemblances et leurs différences (cross-case analysis). L'analyse comparative se fait entre les cliniques rendues à un degré d'informatisation similaire (clinique $\mathrm{A} v \boldsymbol{v}$ clinique $\mathrm{B}$ et clinique $\mathrm{C}$ vs clinique $\mathrm{D}$ ).

Dans le cas des cliniques $E$ et $F$, qui n'avaient pas I'intention de s'informatiser au cours de l'étude, nous dressons un portrait de leur fonctionnement, au début et à la fin de la recherche, et de leurs attentes en ce qui a trait à I'informatisation. La procédure d'analyse est la même que celle expliquée plus haut, mais se limite aux deux premières étapes.

Les gestionnaires sont également interrogés sur les impacts. Les données sont regroupées selon les dimensions étudiées (pattern-matching). L'informa-

7. Yin parle en ces termes: Developing a case description. A second general analytic strategy is to develop a description framework for organizing the case study (p. 101). 
tion recueillie permet de dresser un portrait de leurs perceptions, de leurs craintes et de leurs attentes par rapport aux dimensions choisies.

L'exposé des résultats porte en premier lieu sur les impacts dans les cliniques informatisées et en voie d'informatisation; suivent les attentes exprimées dans les cliniques non informatisées et les impacts perçus par les gestionnaires.

\section{LES IMPACTS DANS LES CLINIQUES INFORMATISÉES}

L'informatisation des données psychosociales, de même que leur intégration au dossier médical, a suivi dans les cliniques A et B sensiblement le même processus. Les intervenantes sociales concernées ont d'ailleurs travaillé conjointement à ce projet informatique.

Les systèmes informatiques mis en place dans chacune des cliniques ont également plusieurs points communs. Dans les deux cas, les données informatisées portent sur le profil ou les caractéristiques socio-démographiques du bénéficiaire, certains tests ou chirurgies effectués ainsi que les rapports synthèses d'évaluation des professionnels de la clinique. Aussi, dans ces mêmes cliniques, les informations relatives au service social sont transmises sous forme de données synthèses codifiées. Ces données sont tirées de la grille informatisée d'intervention familiale et sociale, elle-même intégrée au dossier du service social.

Dans le cas de la clinique $A$, ces données synthèses sont entrées sur I'ordinateur de la clinique et intégrées au dossier médical. De plus, les données complètes de service social sont accessibles pour interrogation et mises au service de la clinique. Après expérimentation, on réalise qu'il y a un problème de programmation, qui empêche l'impression des commentaires accompagnant les données codifiées. Ces données paraissent donc parcellaires et on cherche à y joindre les commentaires contenus sous forme narrative. Des démarches sont entreprises par le directeur de la clinique auprès du service informatique pour corriger cette anomalie. Dans le cas de la clinique $B$, les données synthèses de service social ont pu être entrées sur l'ordinateur de la clinique en janvier 1990 seulement. Des problèmes techniques sont à l'origine de ce délai.

Par ailleurs, les impacts réels observés dans les cliniques $A$ et $B$ depuis I'informatisation sont également similaires. Les effets produits se résument ainsi :

- un accès plus immédiat aux informations se rapportant aux diagnostics des différents professionnels;

- $\quad$ une vue d'ensemble ou une vision globale et intégrée des informations concernant le bénéficiaire; 
- des données plus systématiques, plus concises, plus uniformes et facilement repérables, mais des données plus restreintes ou moins nuancées;

- une diminution du temps accordé aux tâches de bureau, dégageant ainsi plus de temps pour les interventions professionnelles;

- une orientation légèrement différente des tâches d'évaluation des besoins et d'élaboration du plan d'intervention, puisqu'elles doivent tenir compte des données synthèses recueillies;

- des mécanismes de sécurité plus efficaces pour préserver la confidentialité.

Les impacts suscités s'avèrent donc positifs à la fois dans les cliniques $A$ et $B$ et vont dans le même sens que ce qu'ont observé plusieurs auteurs. Ainsi, comme le souligne Barnett (1984), les attentes relatives à l'informatique ne se sont pas toujours réalisées, mais l'impact en ce qui concerne la diminution du temps accordé aux tâches routinières s'est dans la plupart des cas produit. Alberts (1984) a pour sa part constaté que cet allègement laissait plus de temps aux fonctions reliées à l'intervention. Ces observations se confirment également dans le cas des cliniques A et B, tout comme l'accessibilité plus grande aux informations qui, avec l'utilisation de l'informatique, ne fait plus aucun doute (Schoech et Schkade, 1980; Velasquez et Lynch, 1982; King, 1984 ; Moulin, 1986). On croit également que les informations sont aussi bien protégées avec l'informatique et que l'utilisation de celle-ci ne compromet pas la confidentialité. Cette réticence maintes fois rappelée en ce qui regarde la confidentialité semble s'estomper avec le temps (Soussi, 1986; Glastonbury, 1986; Sung, 1982). En un sens, les données informatisées assurent une plus grande confidentialité, puisqu'elles limitent la compréhension du dossier aux seuls initiés et assurent aux membres des cliniques informatisées le contrôle sur la diffusion de l'information.

Par ailleurs, les cliniques $A$ et $B$ fonctionnent depuis plusieurs années en équipe multidisciplinaire; l'informatisation des données cliniques et leur intégration au dossier médical actualisent en quelque sorte cet état de fait. L'informatisation n'aurait pas modifié le rythme et la fréquence des communications entre les membres de ces cliniques. Ces échanges sont jugés très importants en raison des aspects humains qui y sont traités. Au dire de tous, les données informatisées ne sauraient refléter entièrement cette partie de la réalité. La diminution des communications entre les professionnels appréhendée par Moulin (1986) n'a été constatée à la clinique A ni à la clinique B. Les communications établies ne semblent pas avoir été affectées jusqu'à maintenant, à quelque niveau que ce soit. Dans le cas des cliniques A et B, des modes de communication sont établis et maintenus depuis plusieurs années et on ne semble pas vouloir modifier cette façon de faire. En réalité, pour les deux cliniques, l'informatique est vue comme un outil technique de support 
ou encore de référence qui ne saurait modifier des aspects aussi fondamentaux que le suivi professionnel et la relation avec le bénéficiaire. Au contraire, I'informatique doit s'adapter à la pratique professionnelle. II n'en reste pas moins que certains membres de la clinique B en particulier resteraient avec une inquiétude: est-ce que, à plus long terme, les intervenants ne seront pas tentés d'acheminer les données informatisées au détriment des rencontres ou des discussions habituelles?

Tant pour la clinique A que pour la clinique B, le processus d'implantation de l'informatique est apparu long et laborieux. Plusieurs difficultés rencontrées ont trait au financement du système, au choix du matériel et à la conception des programmes. Dans le cas de la clinique A, le processus a été ralenti par l'absence de l'intervenante sociale à l'origine du projet, qui a été remplacée tardivement et partiellement. La remplaçante a dû s'adapter aux besoins de la clinique en même temps qu'elle s'initiait à l'informatique. À la clinique $A$, on indique par ailleurs que le processus d'informatisation a favorisé la concertation et la complémentarité entre les différents professionnels concernés. À la clinique $B$, on souligne que, malgré les problèmes rencontrés, le processus a suscité la créativité et l'innovation. Comme l'explique Guimond et Bégin (1987), le succès de l'implantation d'un système informatique est principalement fonction d'une perception de l'informatique comme élément facilitant l'atteinte des buts de l'organisation ou de ceux des utilisateurs. Enfin, après plusieurs années d'effort, autant dans les cliniques A et B, on se sent essoufflé et moins disposé à entreprendre des démarches visant à étendre le projet à d'autres cliniques ou d'autres organismes.

\section{LES IMPACTS DANS LES CLINIQUES EN VOIE D'INFORMATISATION}

Les cliniques $\mathrm{C}$ et $\mathrm{D}$ tiennent toutes les deux un dossier parallèle au dossier central ou dossier des archives de I'hôpital. Ce dossier propre à chaque clinique se veut plus personnalisé et accessible. Les informations contenues sont sensiblement les mêmes d'une clinique à l'autre. Ces dossiers regroupent à la fois les données sur les caractéristiques du bénéficiaire et celles sur les principales activités de traitement poursuivies. Les informations relatives au service social n'apparaissent cependant pas au dossier de la clinique D. Les informations concernant les résultats de laboratoire sont informatisées à la clinique $\mathrm{D}$ et celles reliées à la médication le sont à la clinique $\mathrm{C}$. En plus de servir à documenter et à soutenir les discussions de cas, le dossier et les informations qui y sont consignées sont très utilisés pour la recherche dans les deux cliniques.

Les intervenantes sociales des cliniques $C$ et $D$ ont expérimenté, à I'intérieur d'une formation donnée par le service social, la grille informatisée d'intervention familiale et sociale. La clinique $\mathrm{C}$ a commencé à informatiser 
certains de ses dossiers. La praticienne de la clinique $\mathrm{D}$, quant à elle, entrevoit des modifications dans le contenu et la tenue du dossier. Elle présume que l'utilisation de la grille changera la façon de rédiger l'évaluation psychosociale et le plan d'intervention qui y seront alors présentés sous forme de données codifiées.

L'intervenante de la clinique $\mathrm{C}$ mentionne que la grille s'applique bien à la pratique sociale de sa clinique et que son utilisation permet de définir les besoins et de décrire l'intervention plus facilement en plus de sauver un temps considérable. Elle estime que la grille informatisée rend tout aussi bien le portrait du bénéficiaire que le rapport narratif. Une seule réserve est soulevée: on peut difficilement faire des nuances lorsqu'on codifie les informations. De son côté, l'intervenante de la clinique $\mathrm{D}$ s'interroge sur l'adaptabilité de l'instrument à la problématique traitée à sa clinique. Pour elle, il s'avère difficile d'établir en service social des unités de mesure communes à toutes les situations rencontrées et à toutes les interventions effectuées.

Les intervenantes des cliniques $C$ et $\mathrm{D}$ assurent, à la suite d'une requête du médecin, un suivi psychosocial auprès du bénéficiaire. Eu égard à la sévérité du diagnostic, les incidences psychosociales sont très marquées dans I'une comme dans l'autre clinique. L'intervenante de la clinique $\mathrm{C}$ voit $\mathrm{d}^{\prime}$ 'abord tous les patients hospitalisés et elle effectue des interventions suivant les problèmes rencontrés. L'intervenante de la clinique $D$ agit principalement lorsque le bénéficiaire retourne à la maison. Elle est alors appelée à jouer un rôle d'agent de liaison et de médiation auprès des diverses ressources du réseau. À la clinique $\mathrm{C}$, on ne croit pas que l'informatisation éventuelle des données psychosociales modifie le suivi auprès du bénéficiaire. L'informatique est vue avant tout comme un outil supplémentaire d'aide à l'intervention.

Par ailleurs, les cliniques $C$ et $D$ favorisent une approche multidisciplinaire. Les équipes respectives se rencontrent de façon formelle pour échanger sur les besoins du bénéficiaire et établir conjointement un plan d'intervention. Des communications régulières et constantes sont maintenues entre les différents professionnels. On estime que ces communications devraient se poursuivre de la même façon avec l'informatisation. À la clinique $\mathrm{D}$, on pense que les données informatisées, plus disponibles et plus accessibles, devraient enrichir ces communications.

En ce qui a trait à la confidentialité, même si dans les cliniques $C$ et $D$ on croit aux moyens techniques de protection, les intervenants continuent à s'interroger sur l'efficacité des mesures de préservation de la confidentialité lorsqu'on utilise l'informatique. On considère que l'accès aux banques de données devrait être contrôlé et qu'on devra s'interroger sur la nature des données à emmagasiner dans l'ordinateur. Bien que les professionnels de la santé et des services sociaux soient confiants quant à la protection qu'apporte I'informatique, Sung (1982) a observé que, paradoxalement, ces derniers 
demeurent sous la hantise de voir la confidentialité compromise soit en principe, soit dans les faits. Les données recueillies dans les cliniques en voie d'informatisation semblent confirmer ces appréhensions.

Les attentes formulées dans les cliniques $C$ et D à l'égard de l'informatisation des données cliniques sont nombreuses et sensiblement les mêmes d'une clinique à l'autre. L'informatisation devrait permettre notamment:

- de préciser et synthétiser les informations concernant le bénéficiaire;

- d'accéder plus rapidement à ces informations ;

- de connaître le profil des clientèles;

- d'avoir une vue d'ensemble des interventions des différents professionnels;

- de démontrer les coûts de ces interventions ou des services offerts ;

- d'économiser du temps dans l'accomplissement des tâches de bureau;

- de diminuer la paperasse;

- de compiler les statistiques;

- d'évaluer et de comparer les résultats;

- d'intervenir avec plus de célérité;

- d'éclairer les décisions professionnelles;

- de favoriser et d'enrichir les communications.

Toutefois, tout en étant plus accessibles, on craint que les données psychosociales soient plus limitées, qu'elles manquent de finesse et de nuance. À cet égard, on s'interroge sur la standardisation et la codification $d^{\prime}$ informations qui doivent refléter des aspects humains.

À la clinique $C$, la priorité en matière d'informatisation a été donnée à la mise sur pied de banques de données à des fins de recherche qui, juge-t-on, est une entreprise plus facile comparativement aux démarches à réaliser pour informatiser les données cliniques. Des raisons semblables sont mentionnées à la clinique D. Des limites de temps et une surcharge de travail empêcheraient les professionnels intéressés au développement $d^{\prime}$ 'un système informatisé des données cliniques de poursuivre les démarches d'implantation. De plus, le départ de l'intervenante sociale de cette clinique, sur qui reposait I'initiative de ce projet, a freiné toute velléité d'implantation immédiate. Certains auteurs (Glastonbury, 1986; Pinkerton et Raffoul, 1984) mentionnent que la charge de travail, le manque de temps et les démarches inhérentes à la mise en place des systèmes sont des arguments invoqués pour ralentir le processus et qu'ils deviennent même parfois des obstacles à l'informatisation. Les observations recueillies dans les cliniques $\mathrm{C}$ et $\mathrm{D}$ le confirment. Les besoins en informatique ne sont pas tout à fait les mêmes dans les cliniques $C$ et D. Dans la clinique $\mathrm{D}$, les besoins prioritaires ont trait à la recherche. 
On souhaite également que les différents formulaires administratifs soient informatisés. Ce deuxième aspect n'est pas mentionné dans la clinique $\mathrm{C}$, où l'informatique devrait en priorité faciliter la recherche.

\section{LES ATTENTES DANS LES CLINIQUES NON INFORMATISÉES}

La clinique E possède son propre dossier, afin d'avoir en tout temps les informations nécessaires au suivi du bénéficiaire. On y trouve des données sur les caractéristiques du bénéficiaire et sur la chronologie des activités de traitement. Le dossier de la clinique $F$, pour sa part, contient des résultats d'examens seulement.

Les intervenantes sociales des cliniques $\mathrm{E}$ et $\mathrm{F}$ gardent également un dossier particulier au service social pour chacun des patients qui leur sont confiés. Les informations qui y sont consignées ont trait à l'évaluation psychosociale, à la chronologie des activités, au plan d'intervention, aux rapports de révision ou autres rapports professionnels pertinents. Le contenu de ce dossier est jugé utile et adéquat. L'intervenante de la clinique $F$ rédige ses dossiers selon les rubriques de la grille d'intervention familiale et sociale. Elle estime que l'évaluation faite à l'aide de cette grille est plus succincte et assure une meilleure confidentialité. On peut donc dire que l'informatisation des cliniques A, B, C et D a eu des effets indirects, au moins dans une clinique qui n'a pas encore opté pour l'informatique. Cette dernière clinique a tendance à utiliser des formules de rapport plus structurées et similaires à celles qu'on trouve dans les dossiers informatisés.

Suivant la référence du médecin et en conformité avec le diagnostic établi, les intervenantes sociales des cliniques $E$ et $F$ interviennent principalement auprès des bénéficiaires hospitalisés. Elles évaluent alors les besoins, élaborent le plan d'intervention, apportent le suivi psychosocial nécessaire ou dirigent vers les ressources appropriées. Ce rôle de référence demande, pour l'une comme pour l'autre, de rédiger et de présenter plusieurs rapports d'évaluation.

Concurremment à ce travail de liaison avec les organismes du milieu, les intervenantes font partie de l'équipe multidisciplinaire de leurs cliniques respectives. Ces équipes se rencontrent formellement à toutes les semaines. À ces rencontres sont discutés des cas problèmes et sont déterminés les objectifs à poursuivre dans I'intervention. Dans le cas de la clinique F, les membres de l'équipe voient ensemble tous les patients hospitalisés. Les évaluations des différents professionnels sont nécessaires aux demandes de référence effectuées par l'intervenante sociale. Dans l'une comme dans l'autre clinique, des échanges constants et continus sont maintenus entre les professionnels. 
En ce qui concerne la confidentialité, des mesures sont prises conformément aux règles en vigueur. À cet effet, les informations portées au dossier ont un caractère général, le moins personnalisé possible. Bien que les mesures de sécurité soient jugées adéquates, on déplore que le bénéficiaire circule dans l'établissement avec son dossier en main, ce qui, selon la perception des intervenantes, peut compromettre la protection des informations.

Rappelons qu'aucun projet d'informatisation n'est en vue dans les cliniques $\mathrm{E}$ et $\mathrm{F}$. Advenant cependant qu'on procède à l'informatisation des données cliniques, certaines attentes sont formulées. On pense que l'informatisation devrait permettre:

- d'alléger les tâches de bureau ou secondaires, dégageant ainsi plus de temps pour les interventions professionnelles;

- de diminuer la paperasse;

- d'avoir une vue globale des besoins du bénéficiaire et des interventions professionnelles effectuées auprès de ce dernier.

À la clinique $\mathrm{E}$, on pense que les communications verbales sont essentielles dans un contexte de pratique où le facteur humain prédomine. On craint aussi que les données, une fois informatisées, perdent de leur valeur qualitative, qu'elles laissent place à l'interprétation et qu'elles compromettent la confidentialité. Ces craintes quant à l'interprétation des données sont aussi partagées dans la clinique F. Dans un contexte de pratique qui demande beaucoup de souplesse et d'adaptabilité et où les données cliniques fluctuent constamment, les membres de la clinique $\mathrm{E}$ s'inquiètent de la rigidité des systèmes informatiques. Dans la clinique $F$, c'est en fonction de l'efficacité et du rendement pour la collecte, l'enregistrement et la transmission des informations qu'on perçoit positivement l'informatisation des données cliniques.

\section{LES IMPACTS VUS PAR LES GESTIONNAIRES}

Le projet d'informatisation des données cliniques soulève des interrogations chez les gestionnaires. Ces interrogations touchent plus directement la codification des données. Ainsi, on se demande si les codes utilisés pour recueillir et transmettre les informations sont suffisamment explicites; si les professionnels concernés s'appliqueront à déchiffrer ces codes; $s^{\prime}$ ils modifieront en conséquence leurs habitudes de travail ; si les ressources ou services extérieurs, à la suite d'un transfert de dossier, comprendront ces mêmes codes. On considère également que les données sociales présentées sous forme de code ne sont pas suffisantes et doivent être détaillées ou expliquées. On croit que les codes utilisés standardisent et uniformisent les informations et ne rendent pas entièrement compte de la réalité d'une problématique sociale. 
Les données codifiées sont par ailleurs vues comme complémentaires à d'autres informations. Elles donnent des indications et, à ce titre, peuvent s'avérer très utiles.

L'informatisation des données cliniques ou de service social est jugée nécessaire pour répondre aux besoins de recherche et d'évaluation des résultats et pour rendre compte à la population des services fournis. On trouve également qu'elle simplifie la rédaction des dossiers, diminue la quantité $d^{\prime}$ informations et facilite la lecture des rapports d'évaluation. De plus, pour les gestionnaires, I'informatisation et l'intégration des données de service social au dossier médical concrétisent la complémentarité, la multidisciplinarité et le décloisonnement des services. Elles donnent aussi plus de visibilité à l'évaluation sociale.

Concernant les mesures de préservation de la confidentialité, deux groupes d'opinions différentes mais complémentaires se dégagent des propos des gestionnaires. D'une part, comme l'informatique favorise une plus grande accessibilité à l'information, il y a lieu de se demander quelles sont les données psychosociales qu'il est essentiel de transmettre et de divulguer. De même, comme plusieurs professionnels peuvent avoir accès à cette information, on croit important d'aviser le bénéficiaire ou de demander son autorisation. Avec la venue du projet «Système intégré des dossiers cliniques » $(\text { Sidoci })^{8}$, on estime que plusieurs règles de sécurité pourront prendre forme d'ici quelques années. D'autre part, on pense que l'informatique peut renforcer la confidentialité parce que les codes utilisés se révèlent plus hermétiques. On juge aussi que les clefs de protection sont suffisamment sécuritaires pour que l'accès aux divers types de données puisse être restreint aux groupes de professionnels immédiatement concernés. On soutient qu'il ne faut pas compromettre l'accessibilité des données psychosociales uniquement en raison de leur caractère hautement confidentiel, mais plutôt adapter les mesures de protection de la confidentialité en fonction des données à transmettre et de leur destinataire.

Les gestionnaires portent un jugement positif à l'endroit du projet d'informatisation qu'ils qualifient par ailleurs d'innovateur. On souligne que ce projet a été conduit par des personnes très motivées et mérite qu'on réfléchisse sur le processus et sa conception. On note l'effort, le souci de recherche et la rigueur des personnes qui en ont fait la promotion. Ce projet paraît particulier à certaines cliniques, à une problématique donnée, et on ne saurait dire si l'expérience pourra être généralisée à d'autres milieux de pratique.

8. Le projet Sidoci, visant cinq hôpitaux dont I'hôpital Sainte-Justine, a débuté en 1987 et avait pour but d'informatiser tout le dossier médical du bénéficiaire, y compris les données psychosociales. 
Par ailleurs, ce qui est perçu plus négativement par les gestionnaires concerne l'implantation et le développement du projet. Lancé par les professionnels eux-mêmes, ce projet démarrait, selon les gestionnaires interrogés, avec un atout important. On considère cependant qu'il aurait fallu que les instances décisionnelles s'impliquent davantage dès le départ. Les demandes de financement des instigateurs ont parfois été perçues comme pressantes, alors qu'il était difficile de défendre un projet qui n'était inscrit dans aucun plan de développement informatique, ni à l'hôpital ni au Centre de services sociaux. Comme ces établissements fonctionnent par priorités et que les projets d'informatisation doivent toucher le plus de secteurs d'activité possible, il s'est avéré difficile de promouvoir un projet qui s'est développé dans un tel contexte. À cet égard, il apparaît essentiel, pour les personnes interrogées, que tout projet informatique soit intégré au projet global de l'organisation.

La mise en place du système informatique pendant sa période de rodage et de transition est perçue également comme étant plus difficile. Le nombre très limité d'ordinateurs a fait en sorte que tout doit être imprimé; les informations sont écrites à la main et introduites dans l'ordinateur par la suite, ce qui occasionne un dédoublement de la tâche. Le fait que deux systèmes se chevauchent alourdit le travail et ne produit pas les effets escomptés.

Finalement, on souhaiterait que le projet évolue, mais le contexte est peu favorable et plusieurs obstacles sont à surmonter. Au moment où a été menée l'étude, la perspective du rapatriement des services sociaux dans les hôpitaux avait un effet de démobilisation et favorisait peu le transfert de I'innovation à d'autres secteurs d'intervention professionnelle. À I'hiver 1991, la démission en bloc du comité informatique formé dans l'unité de service social fait en sorte que bien des projets sont mis en veilleuse.

\section{CONCLUSION}

Somme toute, le bilan est qualifié de positif pour la clinique A et de formateur pour la clinique $B$. Les systèmes mis en place semblent avoir répondu aux besoins d'accessibilité et d'intégration des données cliniques, particulièrement des données psychosociales. Des attentes demeurent: I'utilisation de ces données à des fins de recherche et d'évaluation des résultats, l'amélioration de l'informatisation du dossier du bénéficiaire et l'accessibilité à des terminaux pour les divers intervenants.

II n'est pas approprié de reprendre en détail les constatations mentionnées antérieurement dans l'exposé des impacts observés. Toutefois, deux tendances générales semblent se dégager de cette étude. L'une relève du processus d'informatisation lui-même et l'autre de son impact auprès des usagers actuels et potentiels. 
Sur le plan du processus d'informatisation, la principale observation concerne la participation continue, du début à la fin du processus, de toutes les instances touchées par un projet d'informatisation. Un processus amorcé exclusivement à partir de la base de l'organisation, comme ce fut le cas dans cette expérience, semble entraîner autant d'inconvénients et de résistances que s'il avait été engagé d'autorité par le sommet hiérarchique. La concertation entre les gestionnaires et toutes les praticiennes du service social à partir de la toute première étape du processus semble s'imposer comme modèle à privilégier lorsqu'il s'agit d'implanter un projet d'informatisation dans les services sociaux, à plus forte raison lorsque celui-ci porte sur le contenu de I'intervention des professionnelles. On ne peut pas conclure que le processus soit nécessairement plus efficace lorsqu'il est enclenché uniquement à partir d'un groupe de professionnelles, pas plus qu'il ne le serait sous I'influence exclusive des gestionnaires. On ne peut pas davantage tenir pour acquis que les collègues soient mieux disposés à l'égard d'un projet d'informatisation lancé par leurs pairs qu'à l'égard d'un autre qui serait sous la responsabilité de leurs supérieurs.

Le deuxième aspect concerne les impacts positifs d'un projet d'informatisation. Une constante semble s'en dégager, comme il fut d'ailleurs démontré dans des études précédentes (Béliveau et Poulin, 1987: 141; Poulin et Béliveau, 1987: 69-70; Gandy et Tepperman, 1990: 184), où le contact avec l'informatique devient une variable explicative majeure du développement d'une attitude positive. C'est en effet dans les cliniques déjà informatisées qu'on constate le moins de résistance et le plus d'appréciation positive à l'égard des gains attribuables à l'informatisation. Les craintes et les attentes négatives sont par ailleurs les plus manifestes chez les intervenantes et les gestionnaires qui n'ont pas été directement en contact avec l'expérience d'informatisation. Les observations recueillies dans les cliniques en voie $d^{\prime}$ 'informatisation ou non encore informatisées en témoignent largement. Il en est de même pour plusieurs des commentaires et appréhensions formulés par les gestionnaires des deux établissements.

Bien que plusieurs impacts positifs aient été observés, on peut croire d'ores et déjà qu'au moment où les différents intervenants des cliniques disposeront d'ordinateurs, d'autres changements surviendront dans les habitudes de travail et seront probablement de plus grande importance que ceux observés dans le cadre de cette expérience. Une nouvelle recherche pourrait alors aider à confirmer ou à infirmer cette hypothèse. 


\section{Références bibliographiques}

ALBERTS, F.L. (1984). « Microcomputers in Clinical Practice: Preparing and Involving Office Personnel », dans M.D. Schwartz, Using Computers in Clinical Practice, New York: The Haworth Press, p. 69-73.

BARKER, R.L. (1987). The Social Work Dictionary, États-Unis: National Association of Social Work, 207 p.

BARNETT, G. (1984). "The Computer and Clinical Judgment», dans M.D. Schwartz, Using Computers in Clinical Practice, New York : The Haworth Press.

BEAUdOIN, A., M. POULIN et F. TURGEON-KRAWCZUK (1991). «Regards sur les nouvelles technologies et leur signification pour l'intervention et la formation en service social », Intervention, $\mathrm{n}^{\circ}$ 88, mars : 71-82.

BÉLIVEAU, G.et M. POULIN, en coll. avec G. BEAUdOIN (1991). Informatisation des dossiers de service social en milieu hospitalier: processus d'implantation et impacts. Tome I: Cadre conceptuel et méthodologie de la recherche. Laboratoire de recherche, École de service social, Université Laval, juillet, 96 p.

Béliveau, G.et M. Poulin, en coll. avec G. BEAudoIn (1991). Informatisation des dossiers de service social en milieu hospitalier: processus d'implantation et impacts. Tome II: Processus d'implantation de l'informatique. Laboratoire de recherche, École de service social, Université Laval, juillet, 103 p.

BéliveAu, G., M. POUlin en coll. avec G. BEAudoin (1991). Informatisation des dossiers de service social en milieu hospitalier: processus d'implantation et impacts. Tome III : Impacts de l'informatisation. Laboratoire de recherche, École de service social, Université Laval, juillet, 73 p.

BéliveAu, G., M. Poulin en coll. avec G. BEAUdOIN (1987). Les perceptions et les attitudes des intervenants sociaux du Québec au sujet de l'utilisation de I'informatique dans leur milieu de travail. Recherche subventionnée par le Conseil de la recherche en sciences humaines du Canada (CRSH). Université Laval, École de service social, juillet, 204 p.

Crozier, M. et E. Friedberg (1977). L'acteur et le système, Paris : Éditions du Seuil.

DANZIGER, J., N. KRAEMER, et L. KeNNETH (1986). People and Computers, The Impacts of Computing on End Users in Organizations. New York: Columbia Univ. Press, $268 \mathrm{p}$.

DıON, G. (1986). Dictionnaire canadien des relations du travail. Sainte-Foy: Presses de l'Université Laval, 2e édition, 993 p.

GANDY, J.M. et L. TePPERMAN (1990). False Alarm: the Computerization of Eight Social Welfare Organizations. Wilfrid Laurier Univ. Press, 220 p.

Glastonbury, B. (1986). «Managing the Social Services Computer Systems, Some Problems and Pitfalls», Computer Applications in Social Work and Allied Professions, vol. 3, $\mathrm{n}^{\circ} 1$.

GUIMOND, S. et G. BÉGIN (1987). Le choc de l'informatique, les répercussions psychosociales et le rôle des attitudes, Monographie de psychologie, SainteFoy: Presses de l'Université du Québec, 88 p.

HAMELIN-BRABANT, L. (1987). L'informatisation et l'adaptation au changement: la pratique médicale au CHUL. Thèse de maîtrise, Université Laval.

KING, M.A. (1984). "Micro Computer Applications in a Hospital Social Work Department », dans M.D. Schwartz, Using Computers in Clinical Practice, New York: The Haworth Press, p. 309-315. 
LARSEN, J. (1987). «Implementing Computers in Mental Health Settings », Computers in Human Services, vol. 2, $\mathrm{n}^{\text {os }} 3 / 4: 145-170$.

MOULIN, B. (1986). «Les systèmes de base de connaissance dans les organisations », L'ingénieur, sept.-oct. : 21-27.

Pinkerton, G. et P. RAfFoul (1984). «Professional Colleagues: Confronting the Attitude of Professionals Toward Microcomputers », dans M.D. Schwartz, Using Computers in Clinical Practice, New York : The Haworth Press, p. 61-66.

Poulin, M., A. Beaudoin, G. Béliveau, A. Lamarre et F. Turgeon-Krawczuk (1991). Les effets et l'appréciation de l'utilisation d'un logiciel interactif par les parents d'enfants atteints de fibrose kystique. Laboratoire de recherche, École de service social, Université Laval, nov., 182 p.

POUlin, M. et G. BéLIVEAU (1987). L'utilisation et le développement de l'informatique dans les services sociaux. Synthèse critique $\mathrm{n}^{\circ} 27$, Commission d'enquête sur les services de santé et les services sociaux, Québec : Les Publications du Québec.

SChOECH, D., L. SChKADE et C. HOPPER-Russel (1980). "Computer Helping Caseworkers: Decision Support System », Child Welfare, vol. 59, no 96 : 566-575.

SousSI, A. (1986). La révolution informatique et la pratique professionnelle, Office des professions du Québec, $200 \mathrm{p}$.

SUNG, H. (1982). "Working under the Accountability System: A Study of Reaction of Social Workers », Administration in Social Work, vol. 6, $\mathrm{n}^{\circ} 4$ : 15-30.

TIMMS, N. et R. TIMMS (1982). Dictionary of Social Welfare. Londres : Rutledge and Kegan Ltd.

VelasQueZ, J. et M. LYNCH (1983). "The Practical Use of Microcomputers in Rehabilitation », Rehabilitation Literature, vol. 44, $\mathrm{n}^{\text {os }} 3 / 4: 66-70$.

YIN, Robert K. (1984). Case Study Research: Design and Methods. Beverly Hills: Sage Publications, $159 \mathrm{p}$. 